\title{
Rheology of confined granular flows
}

\author{
Patrick RICHARD*, Alexandre Valance*, Jean-François Métayer*, Jérome \\ Crassous*, Michel Louge ${ }^{\dagger}$ and Renaud Delannay* \\ *Université Rennes 1, Institut de Physique de Rennes, UMR CNRS 6251, 263 av. Général Leclerc, 35042 Rennes \\ cedex FRANCE \\ ${ }^{\dagger}$ Sibley School of Mechanical and Aerospace Engineering, Cornell University, Ithaca, NY 14853, USA
}

\begin{abstract}
The properties of confined granular flows on a heap are studied through numerical simulations and experiments. We explain in details how such kind of flow can be numerically studied using periodic boundary conditions in the direction of the flow. The packing fraction and velocity profiles are found to be described by one length scale. The tracking of the grains show that they exhibit creeping and intermittent cage motion similar to that in glasses, causing gradual weakening of friction at the walls.
\end{abstract}

Keywords: <Enter Keywords here>

PACS: <Replace this text with PACS numbers; choose from this list: http://www.aip..org/pacs/index.html>

\section{INTRODUCTION}

Surface flows of granular materials are frequently observed in industrial processes and in nature. In such type of flows, the moving particles appear to be limited to a surface layer, with a "frozen" bulk region below. They are strongly influenced by sidewalls [1,2]. Interestingly, the gas, liquid and solid behaviors of granular systems are present at the same time in such flows. It is thus the ideal type of flow to test existing theories or to inspire new ones which aim is to describe and predict the whole behavior of flowing granular matter. Most studies concerning these flows have been conducted in two different configurations. The first one is a rotating drum $[3,5,4,6]$, a cylinder, partly filled with granular material, rotating around its axis at a controlled speed. Stationary states can be studied in such kind of configuration but it does not permit fully developed flows (ie flows that are invariant in the main flow direction). The second configuration consists in pouring grains at a constant flow rate between two parallel sidewalls separated by $W[7,1]$. In this configuration a static heap slowly forms by trapping grains at its top. After a transient, the growth of the heap stops and the flowing layer at its surface reaches a steady state. Studying this configuration, Taberlet et al. [1] reported the "side-wall stabilized heap" (SSH) regime, where sidewalls of effective friction $\mu_{w}$ decrease the shear stress in the flow. Using a balance of momentum for a mobilized layer, one can derive an approximate linear scaling law linking the free surface angle, $\theta$ the height of the layer $h$ and the width of the channel, $W$ :

$$
\tan \theta=\mu_{i}+\mu_{w} \frac{h}{W},
$$

where $\mu_{i}$ and $\mu_{w}$ are effective friction coefficient [1]. Thus, confining walls plays a major role in the momentum balance if the second term on the right in this equation dominates over the first. This equation also points out that the relevant parameter to evaluate the effect of the wall friction on the flow is $h / W$ and not $W / d$.

It has been shown that "frozen" bulk layer below the flow is not purely static but exhibit slow creeping motion [8, 9]. Thus this region is in fact a quasistatic layer.

Equation 1 can be derived for a slab parallel to the free surface at depth $y$ and of thickness $d y$ :

$$
S(y) / N(y)=\tan \theta-\mu_{w} y / W .
$$

Assuming that the coefficient $\mu_{w}$ does not depend on $y$, this equation leads to a paradox: the effective friction $S / N$ reverses sign at finite depth.

In this paper we report numerical and experimental results on the rheology of confined granular flows between two vertical sidewalls. We will show that it is possible to simulate such kind of flows using periodic boundary conditions. Then, we will report that the effective sidewall friction coefficient depends on the depth. In doing so, we will resolve 


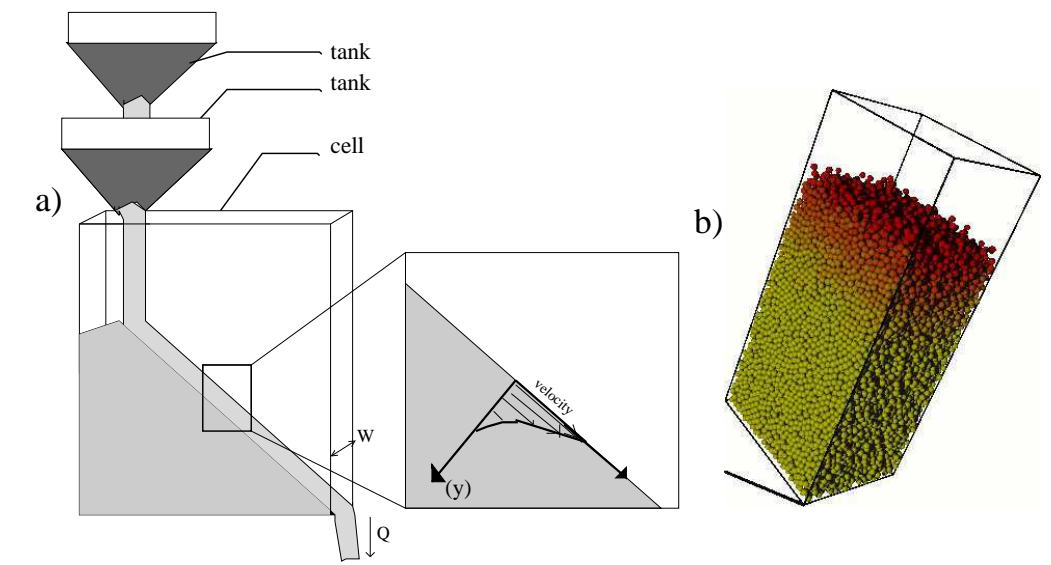

FIGURE 1. Sketch of the apparatus, closed bottom and left, and bound by two plane, parallel, frictional sidewalls (a) and a typical 3D snapshot for chute flow: $N=24,000$ grains, with $W / d=20$, angle of inclination $\theta=35^{\circ}$, coefficient of restitution $e=0.88$, and friction coefficient $\mu=0.50$ (b). The color of the grains corresponds to the velocity in the stream wise direction : $v_{x}$. It varies from yellow $\left(v_{x}=0\right)$ to red (maximum value of the velocity).

the above mentioned paradox.

The outline of this paper is the following. The next section is devoted to the description of our experimental set-up and of the numerical methods. The third section deals with the study of the velocity and packing fraction of these flows. Then, we will report the evolution of the sidewall friction with the depth. The conclusion and some perspectives will be presented in the last section.

\section{EXPERIMENTAL AND NUMERICAL METHODS}

\section{Experimental set-up}

Our experimental set-up (Fig. 1) is similar to the one used by Taberlet et al. [1]. It consists of two $1200 \mathrm{~mm} \times$ $1200 \mathrm{~mm}$ parallel and vertical glass plates separated by width $W$. Glass-beads of diameter $d=(500 \pm 100) \mu \mathrm{m}$ are continuously poured between these plates through a "double-hopper". The lower hopper is continuously filled with particles by the upper one. The aperture of the lower hopper precisely controls the input flow rate $Q$, defined as the mass of material entering the channel per unit of time and per unit of width. In such a set-up, $Q$ and $W$ are the only control parameters. When the system reaches a steady-sate, the input flow rate is equal to the output flow rate which is measured using an electronic scale weighting the material falling out of the channel. All our experiments are conducted in a temperature and humidity-controlled room $\left(20^{\circ} \mathrm{C}\right.$ and $50 \%$ of humidity). The electrostatic effects are minimized by passing the grains through a metal sieve connected to the ground prior to all experiments. The beads are painted in black to limit light reflection. A Photron APX RS camera of $1024 \times 1024$ resolution for 50 to $30,000 \mathrm{~Hz}$ frame rate tracks rapid grains, a Nikon D200 reflex camera at 12 images/minute is used for slow grains. This range of frequency is large enough to track grains whose velocity is between $3.10^{-4} \mathrm{~m} / \mathrm{s}$ and $10 \mathrm{~m} / \mathrm{s}$ (fast camera) and between $3.10^{-2} \mathrm{~m} / \mathrm{s}$ and $1.10^{-6} \mathrm{~m} / \mathrm{s}$ (camera). The bead size is about 30 pixels. An own-made tracking program deduces the velocity of a given grain from the difference between its successive positions, assuming that the tracked grains motion is less that one radius between two images. To estimate the precision of our measures, and the smallest velocity we can detect, the position of grains in an immobile packing have been tracked. The displacements measured are artifacts due to spot light variations inducing fluctuations in the gray level of each pixel. The minimum average velocity that can be detected is then $f / 200 \mathrm{~mm} \cdot \mathrm{s}^{-1}$ where $f$ is the frequency of the camera. The angle of inclination of the flow, $\theta$ is also measured by image processing.

The packing fraction is deduced from the absorption of $\gamma$-rays by the granular material. The channel is placed between the source of $\gamma$-rays (Cs137) and a scintillator which measures the intensity of the beam. This intensity follows the Beer-Lambert law: $I(y)=I_{0} \exp (-\alpha W v(y))$, where $I_{0}$ is the intensity measured when the channel is empty, $\alpha$ the absorption constant which depend on the material ( $\alpha=0.188 \mathrm{~cm}^{-1}$ for glass), $W$ the channel width and $v$ the packing 
fraction averaged in the direction $z$ perpendicular to the sidewalls. More details on the this methods can be found in $[10,11]$.

\section{Numerical methods}

Computer simulations have turned out to be a powerful tool to investigate the physics of granular flow, especially valuable as they reveal flow details, such as stresses, which experiments cannot provide. A very popular simulation scheme is an adaptation of the classical Molecular Dynamics technique [12]. It consists of integrating Newton's equations of motion for a system of "soft" grains starting from a given initial configuration. This requires giving an explicit expression for the forces that act between grains. Let us consider two overlapping spheres $i$ and $j$. The overlap $\delta$ leads to a normal force $F_{n}=k_{n} \delta-\gamma_{n} \dot{\delta}$, where $k_{n}$ is a spring constant, $\gamma_{n}$ a viscous damping. The damping is used to obtain an inelastic collision. The tangential force used is that of the well-known regularized Coulomb law $F_{t}=\min \left(\mu F_{n}, k_{t} u_{t}\right)$ where $\mu$ is friction coefficient, $k_{t}$ a tangential spring stiffness, $u_{t}$ is the tangential displacement of the contact. The latter is set to zero at the initiation of a contact and its rate of change $d u_{t} / d t$ is equal to the sliding velocity. Note that the rigid body motion around the contact is taken into account to ensure that the tangential displacement is always in the local tangent plane of the contact. For most of the results presented here, the following values of the parameters are used: particle diameter $d=0.5 \mathrm{~mm}$ (with a small uniform polydispersity $\pm 20 \%$ to prevent crystalization), density $\rho=2500 \mathrm{~kg} \cdot \mathrm{m}^{-3}, \mathrm{kn}=5.6075 \times 10^{6} \mathrm{mg} / \mathrm{d}$, where $\mathrm{m}$ is the mean mass of a grain, $\gamma_{n}=130.89 \mathrm{~m} \sqrt{\mathrm{g} / d}, k_{t}=2 k_{n} / 7$ and (unless specified) $\mu=0.5$. These values lead to a restitution coefficient $e=0.88$. When studying the effect of the coefficient of restitution, $k_{n}$ is kept constant and $\gamma_{n}$ is tune to obtain the chosen value of $e$. Impacts against the sidewalls are treated as collisions with a sphere of infinite mass and radius, which mimics a large flat surface. Note that the value of friction coefficient and the value of the coefficient of restitution are the same for the wall-grain and the grain-grain interactions.

The use of periodic boundary conditions for flows on a rough bottom and without sidewalls is straightforward: the angle of the free surface is equal to the angle of inclination of the rough bottom. This is no more the case for confined flows on a heap, since the angle of the free surface depends on the input flow rate. Two kind of simulations can be performed:

- A "full system" simulation where the whole system is simulated from the input of the grains to the output. A constant number flow rate is imposed and, as in experiments, above a critical value [1], grains get trapped underneath the flowing layer, and the SSH grows slowly [13] until its free surface reaches a steady angle.

- A periodic boundary conditions simulation, the angle of inclination is set to a given value. The flow rate $Q$ may evolve toward a constant value.

These two simulations are therefore fundamentally different. The "full system simulation" is the closest to the experiments : the flow rate is imposed and the angle of inclination evolves toward a steady value. In the case of the PBC simulation, the angle of inclination is imposed and the flow rate evolves toward a steady value. Interestingly both types of simulation give the same $\tan \theta$.vs. $Q$ curve [14] and all the flow properties are found to be very close [15]. Surprisingly, in the periodic system, grains below the flowing layer remain nearly immobile despite the steep inclination. Thus, the confining walls permit the establishment of a stable equilibrium on the erodible heap at an angle far exceeding what is observed without them. Nonetheless, above an angle $\theta_{\max }$, grains accelerate, and, below $\theta_{\min }$, they come to rest. These critical inclinations of the flowing layer are functions of simulation parameters. Remarkably, they also depend on the relative channel width $(W / d)$, even though the grain diameter $d$ does not appear explicitly in Eq. (1). An important point is that they $\theta_{\max }$ also depends on the number of grains used in the simulation. This point will be addressed latter. In both systems, $Q$ is measured by counting the number of grains flowing through a surface perpendicular to the confining walls per unit time. Note that, in periodic simulations, we observed that flow properties became insensitive to the domain length $L$ along the stream wise direction when $L>10 d$. Therefore, all the periodic simulations were performed using $L=25 \mathrm{~d}$. 

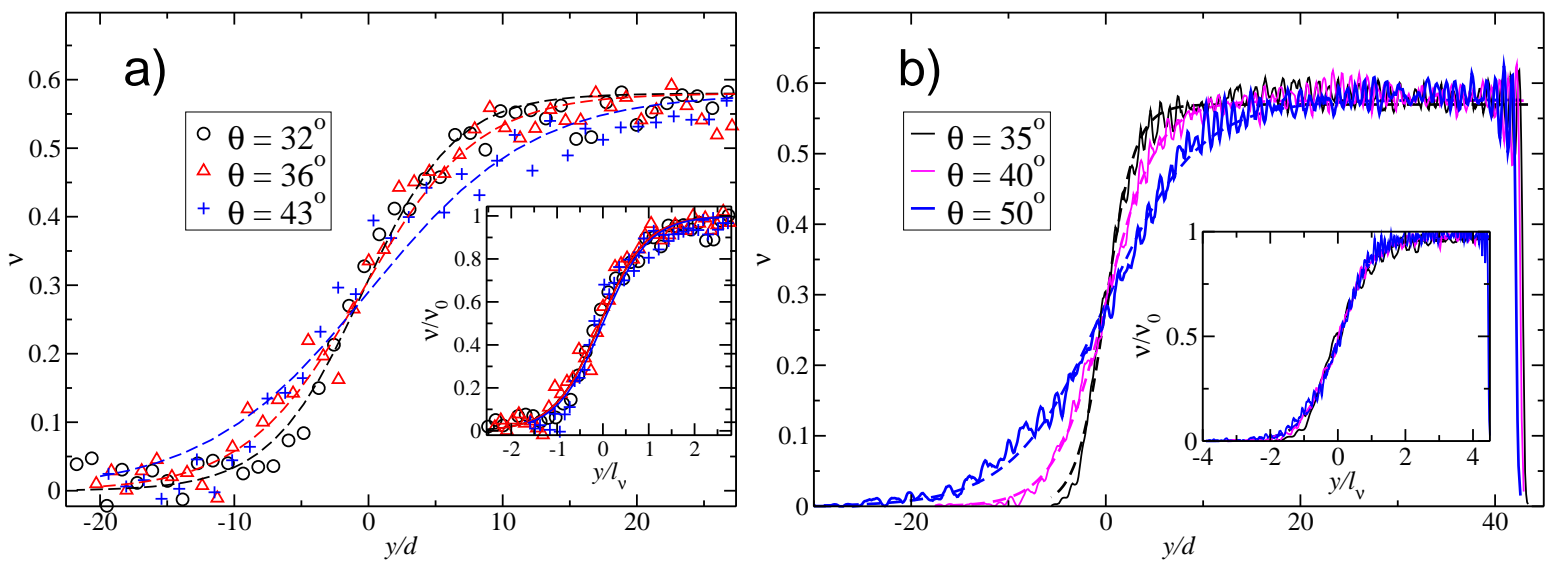

FIGURE 2. Profiles of the packing fraction $v$ for different inclinations shown versus depth for experiments $W / d=18$ (a) and numerical simulations with $W / d=10$ (b). The two insets show how $v / v_{0}$ versus $y / l_{v}$ collapse on a master curve.

\section{PACKING FRACTION AND STREAM-WISE VELOCITY}

\section{Packing fraction and velocity profiles}

Figure 2 shows profiles for the packing fraction $v$ along the downward direction $y$ perpendicular to the free surface. Two distinct regions can be clearly identified: a quasi-static region with a packing fraction approaching 0.58 , toped by a flowing layer where the volume fraction decreases drastically as one approaches the free surface. The packing fraction profiles can be very well fitted by an exponential function of the following form:

$$
v(y)=\frac{v_{0}}{2}\left[1+\tanh \left(y / l_{v}\right)\right]=\frac{v_{0}}{1+\exp \left(-2 y / l_{v}\right)},
$$

where $v_{0}$ and $l_{v}$ are fitting parameters corresponding respectively to the packing fraction in the quasi-static region and to the characteristic length scale over which the packing fraction varies. Note that the origin of the $y$ axis has been chosen such that $v(y=0)=v_{0} / 2$. The two parameters $v_{0}$ and $l_{v}$ completely characterize the packing fraction profiles. If the $y$ coordinate is made dimensionless using the length scale $l_{v}$ instead of the grain diameter $d$, all the curves collapse on a master curve which is nothing but a tangent hyperbolic function. We find that the packing fraction in the quasi-static region is independent of the inclination angle and equal to $v_{0}=0.58$, while the length scale $l_{v}$ increases linearly with the tangent of the inclination angle (Fig. 3):

$$
\frac{l_{v}}{W}=\eta \times\left[\tan \theta-\tan \theta_{0}\right],
$$

with $\theta_{0} \approx 20^{\circ}$. We obtained the same fits from the numerical simulation results, with similar values of the fitting parameters [16]. Equation (3) is thus equivalent to the simple relation between the flow inclination and the relative height $h / W$ of the flowing layer:

$$
\tan \theta=\tan \theta_{0}+\mu_{w}(h / W),
$$

where $\tan \theta_{0}$ and $\mu_{w}$ are interpreted respectively as an internal friction angle of the granular material and an effective friction coefficient at the side walls that accounts for rolling or sliding contacts [1].

The vertical velocity profiles are reported in Figure 4 for experiments (a) and numerical simulations (b). Velocity profiles in the flowing layer share the same length scale $l_{v}$ and all the curves collapse on a single curve $v_{x} /\left(2 l_{v} \sqrt{g / d}\right)$ .vs. $y / l_{v}$. Some differences are observed for small angles of inclination corresponding to flows that are close to jamming. The shear rate is nearly constant in the interval $-1<y / l_{v}<1$. Beneath it, $v_{x} /\left(2 l_{v} \sqrt{g / d}\right)$ smoothly transitions into a decaying exponential. Deeper, as experiments in the quasistatic region will show, creeping velocities drop on a shorter scale $[8,9,16]$. Other works report that the shear rate is no more constant in the case of large channels for rotating drum [6] and confined chute flows [2].

Interestingly, the packing fraction $v$ and the stream wise velocity $v_{x}$ share the same characteristic length $l_{v}$. An 

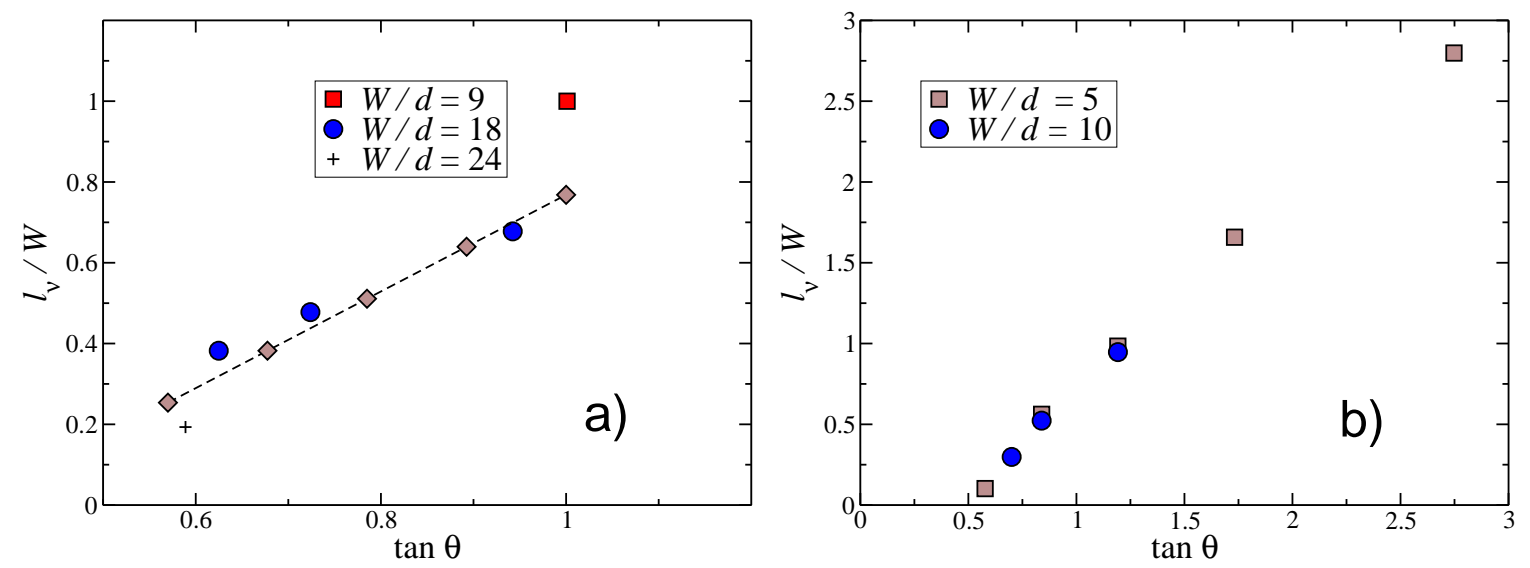

FIGURE 3. Variations of the scale $l_{V}$ with $\tan \theta$ for experiments (a) and numerical simulations (b).
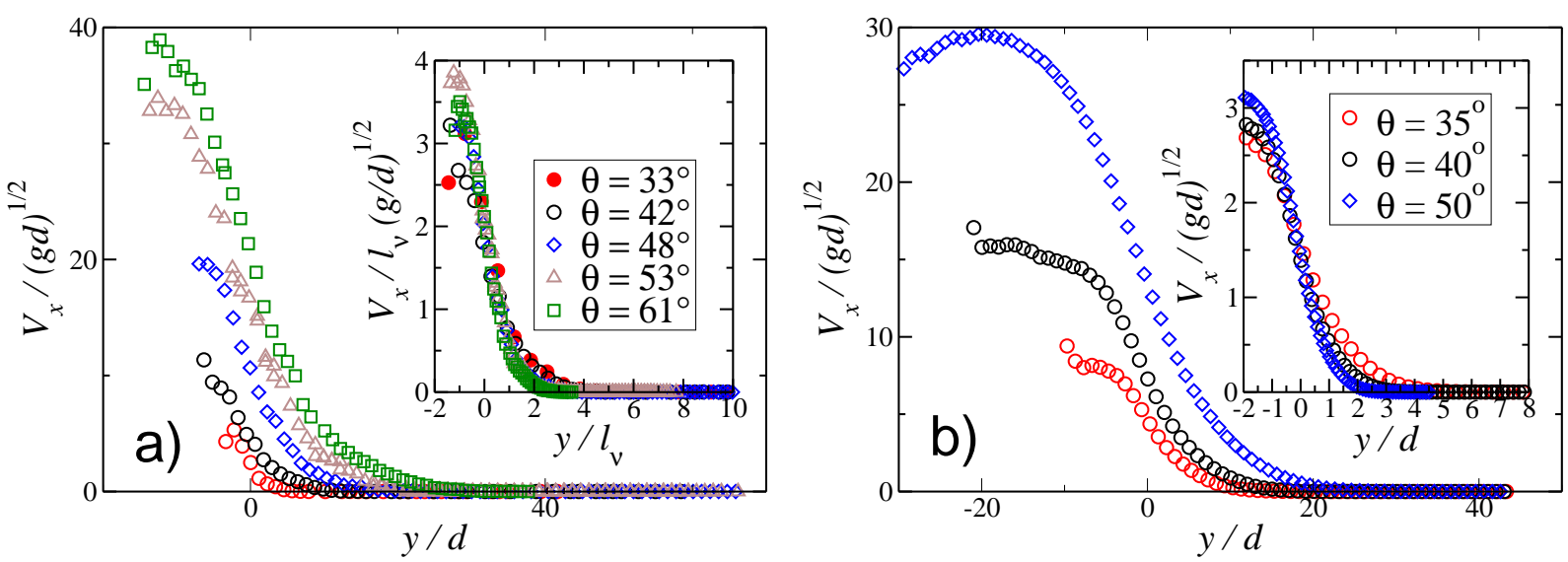

FIGURE 4. Profiles of stream wise velocity $V_{x} / \sqrt{g d}$ for experiments with $W / d=9$ (a) and numerical simulations with $W / d=10$ (b). The insets shows how profiles collapse in the flowing layer if velocity and distance are made dimensionless, respectively, with $l_{v}$ and the characteristic speed $V_{0}=2 l_{v}(g / d)^{1 / 2}$.

important point is that both velocity and packing fraction are significantly different to what is observed for flows on a bumpy bottom [17]. In this latter case the packing fraction is found be constant within the flow which is not the case for confined flows. Note that this difference may be a consequence of the steeper angles of inclination needed to observe confined granular flows. The differences on velocity profiles are even more dramatic. For flows on bumpy bottom the velocity profiles are concave whereas, for flows on a heap, the profiles are convex. This dramatic difference stress ou the importance of the boundaries (rough and bumpy .vs. erodible "bottom") and of the confinement in granular flows.

\section{Effect of the number of grains}

The numerical results reported in figures 2, 3 and 4 have been obtained using 12,000-grains simulations with $\mathrm{W} / \mathrm{d}$. This number of grains is large enough to form a quasi-static layer atop which the flow occurs. As reported in previous section, in the PBC simulations, the input parameters are the gap between the sidewalls and the angle of inclination. Another parameter is the number of grains used for the simulation : $N$. Let us assume that this is number is large enough to form the quasistatic layer. Do the properties of the flow depend on this number, ie on the height of the quasitatic layer? To address this question we report on Figure 5 the variation of packing fraction, stream-wise velocity and velocity fluctuations profiles for a large range of $N$ (from $N=1,000$ to $N=6,000$ ), for $W / d=5$ and $\theta=40$. 

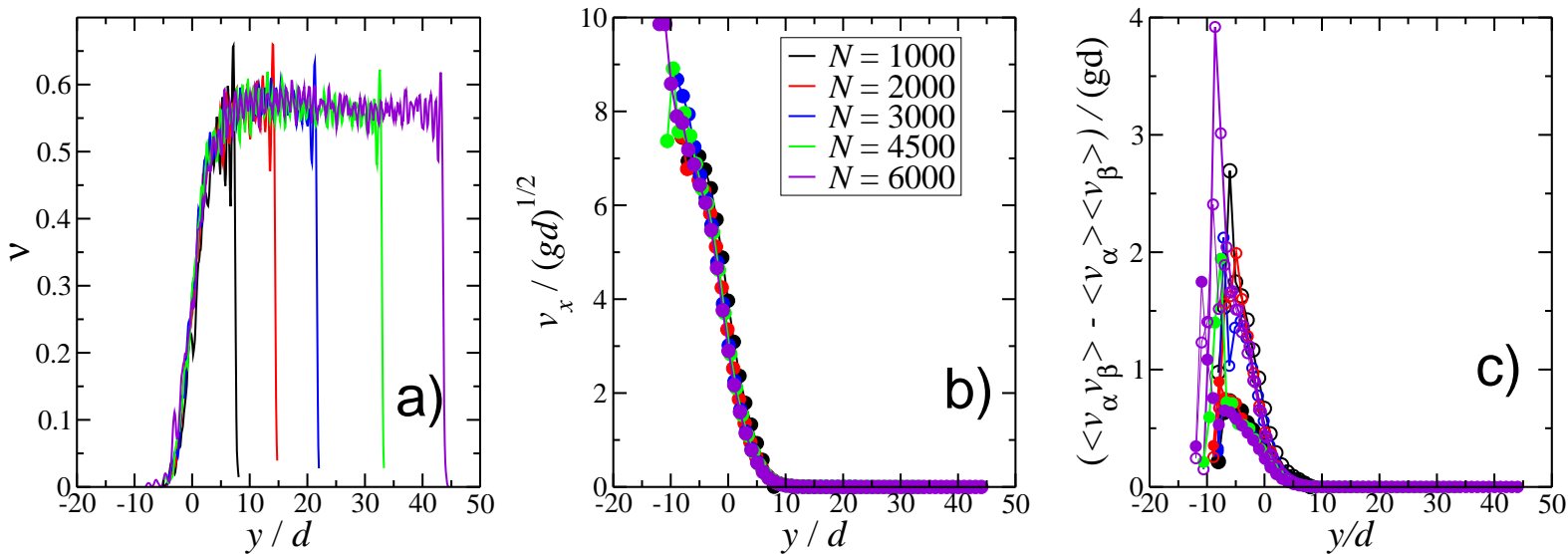

FIGURE 5. Effect of the number of grains used in the PBC simulation with $W / d=5$ and $\theta=40^{\circ}$ on (a) the packing fraction profiles, (b) the stream-wise velocity profile and (c) the velocity fluctuation profiles (c). In the latter, open symbols : $\alpha=x, \beta=x$, full symbols $\alpha=y, \beta=y$.
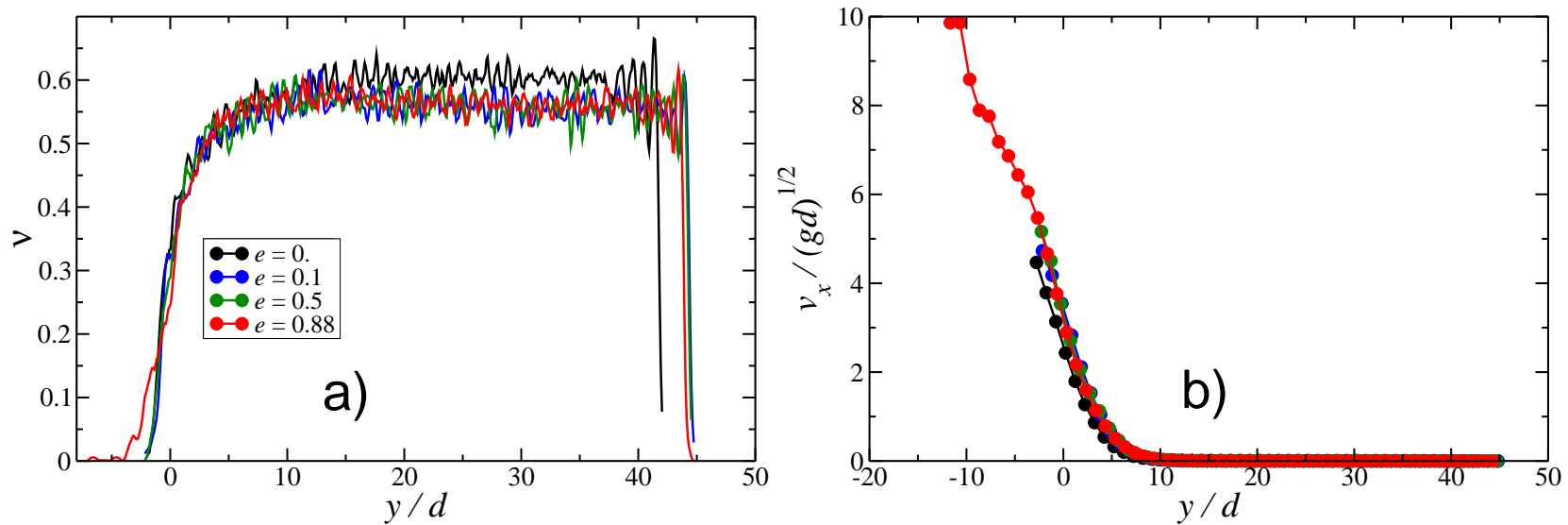

FIGURE 6. Vertical profiles of the packing fraction (a) and of the velocity (b) for different coefficients of restitution. The gap between sidewalls is $W / d=5$, the friction coefficient $\mu=0.5$ and the angle of the flow is $40^{\circ}$.

All the curves collapse, except -of course- in the quasistatic layer which height depends on $N$. This demonstrates that the properties of the flowing layer depend very weakly on $N$ if this number of grains is large enough to create a basal quasistatic layer. This means that the interactions between the flowing and the quasistatic layers are limited to a very short distance. As reported in $[7,8,9]$ the velocity profile in the quasistatic region decreases exponentially. Thus, it is tempting to speculate that this short distance corresponds to the characteristic length of this decay which is of the order of magnitude of the grain size.

The effect of the number of grains on the properties of the quasistatic layer will be discussed in another paper. Note that although the reported results are obtained for a specific configuration $\left(W / d=5\right.$ and $\left.\theta=40^{\circ}\right)$ the conclusions reported generic to all the values of $\theta$ and $W / d$ we have used.

\section{Effect of micromechanical parameters}

In this subsection we report the influence of two micromechanical parameters: the coefficient of restitution and the friction coefficient. These two coefficient have dramatic effects on the collision between two grains but do they affect significantly the macroscopic properties of the flow 

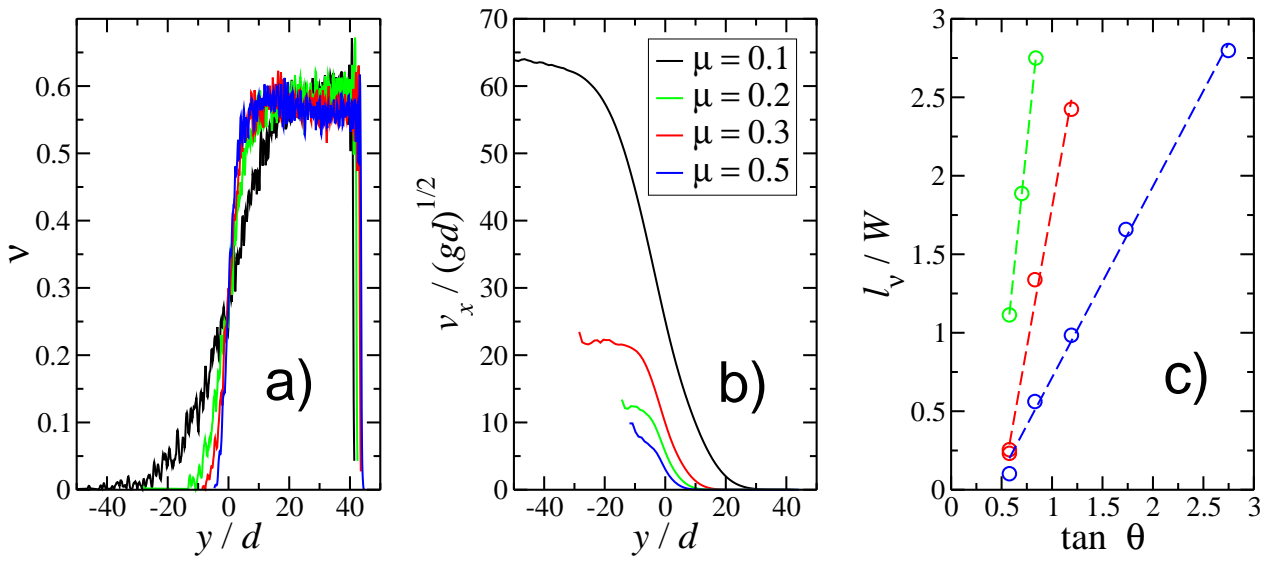

FIGURE 7. Vertical profiles of the packing fraction (a) and of the velocity (b) for different friction coefficients. The evolution of the characteristic length $l_{v}$ with the packing fraction with the angle of inclination is reported in (c).

Effect of the coefficient of restitution. Figure $6 \mathrm{a}$ and figure $6 \mathrm{~b}$ report respectively the velocity profiles in the direction of the flow and the packing fraction profiles for different values of the coefficient of restitution. We explore a large range of coefficient of restitution from $e=0$ to $e=0.88$. The figures show that variation in the coefficient of restitution has little effect on the flow behavior. Among the differences, we can observe that:

- the packing fraction of the quasistatic layer is slightly higher for $e=0$.

- the thickness of the gaseous layer (where the grains describe ballistic trajectories) increases with $e$.

These weak differences are consistent with what has been observe for flows on a bumpy bottom [17] where the effect of $e$ is also found to be very little. The weak effect of the coefficient of restitution can be understood in terms of non-local dissipation. As reported by Rajchenbach [19], in granular flows, a nearly infinite number of interactions occur in a finite time. Thus the kinetic energy of a grain colliding the flow is attenuated very rapidly. Consequently, the grain-grain coefficient of restitution $e$ has a very little effect since the collisions are virtually almost completely inelastic. This is why the observed rheology appears almost independent of the real elastic restitution coefficient $e$ of the grains.

Effect of the coefficient of friction. Another microscopic parameter we have explored is the friction coefficient. We tune this coefficient from 0.1 to 0.5 . As mentioned in the method section the friction coefficient is the same for the grain-grain and for the grain-wall interactions. Contrary to the coefficient of restitution, this parameter has an important effect on the flow properties (see Fig. 7). Decreasing the friction coefficient increases the characteristic length $l_{v}$. As expected decreasing this coefficient also increases the velocity and the shear rate. Note that these results are somewhat different from what is observed for granular flows on a bumpy bottom [17]. In such a system, the friction coefficient has little effect on the packing fraction. This confirms the importance of dissipation by friction in confined granular flows. The next step of this study is to tune independently these two friction coefficients to point out precisely their relative importance on the flow properties.

\section{FRICTION WEAKENING}

Unlike earlier studies [1,2], which treated $\mu_{w}$ as a constant, our simulations show that the resultant $\mu_{\tau}=\left|\overrightarrow{\tau_{w}}\right| /\left|\sigma_{z z}^{w}\right|$, which we compute as the magnitude ratio of the surface force $\overrightarrow{\tau_{w}}=\sigma_{z z}^{w} \overrightarrow{e_{x}}+\sigma_{z y}^{w} \overrightarrow{y_{y}}$, and normal stress $\sigma_{z z}^{w}$ on sidewalls, weakens with depth, as shown in figure 8 for different inclinations. Close to the free surface, $\mu_{\tau}$ is close to the graingrain friction coefficient and varies little. At greater depths $\mu_{\tau}$ decreases sharply. Here again, $\mu_{\tau}$.vs. $y / l_{v}$ collapses on a master curve except for angles corresponding to flows close to the jamming transition (see insets of fig 8).

A full characterization of sidewall friction requires the knowledge of its direction. To address this point we record in our simulations the direction $\phi$ of the wall friction $\overrightarrow{\tau_{w}}=-\left|\overrightarrow{\tau_{w}}\right|\left(\cos \phi \overrightarrow{e_{x}}+\sin \phi \overrightarrow{e_{y}}\right)$, which varies with $y / l_{v}$. An angle $\phi$ equals to zero means that the direction of the friction is the opposite of the main direction of the flow. In the flowing 

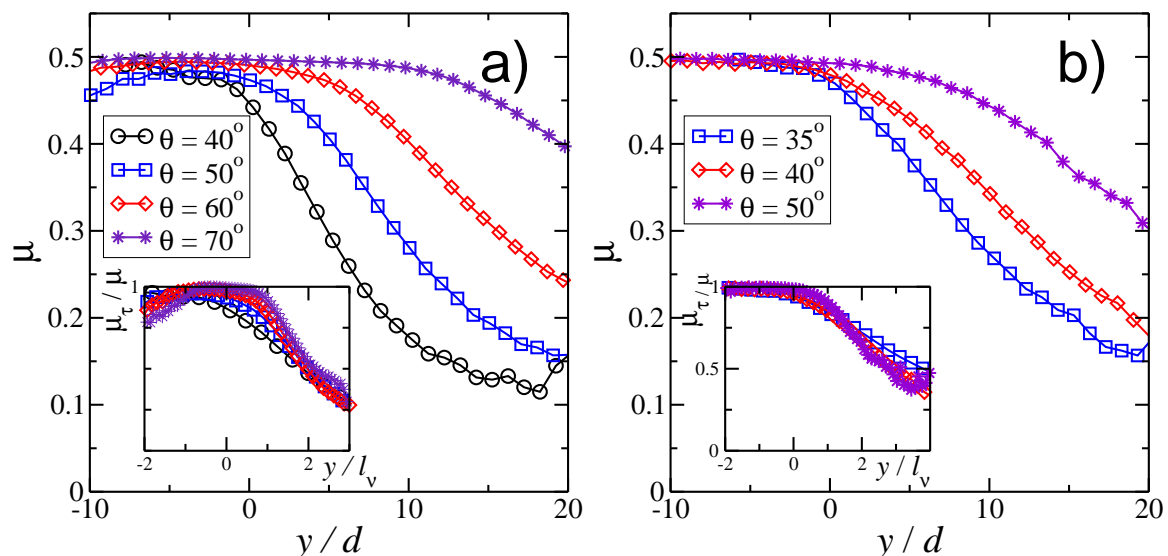

FIGURE 8. Variation of the resultant sidewall friction $\mu$ with depth for several $\theta$ for $W / d=5$ and $W / d=10$. The inset reveals a master curve of $\mu_{\tau} / \mu$.vs. $y=l_{v}$ which becomes linear for $y / l_{v}>2$ (dashed line).
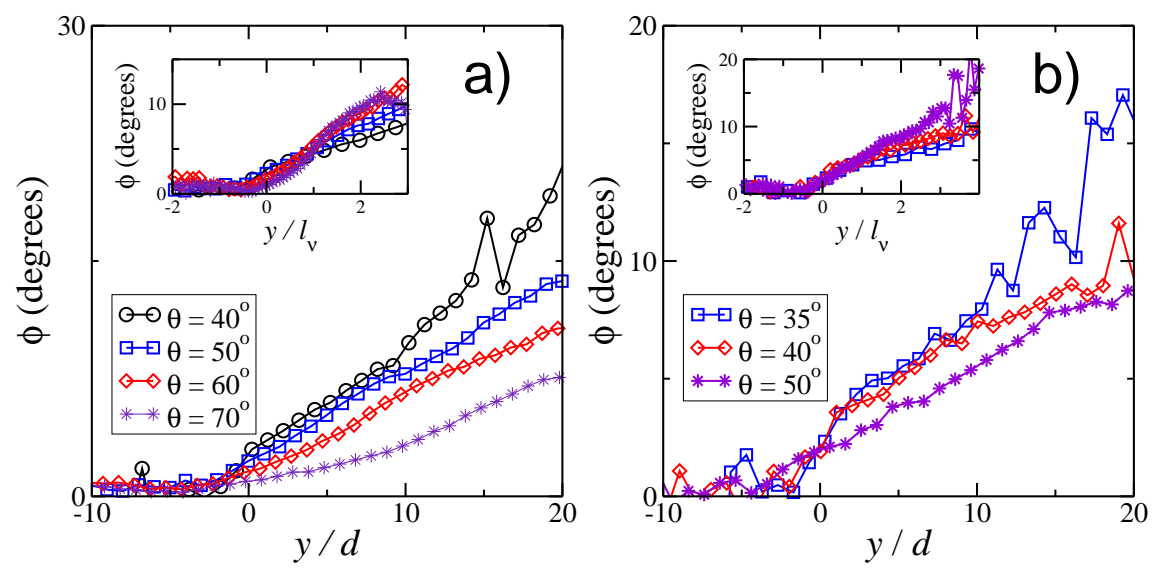

FIGURE 9. Variation of the angle of the resultant sidewall friction $\phi$ with depth for several $\theta$ and for $W=5 d$ (a) and $W=10 d$ (b). The inset reveals a master curve of $\tan \phi$.vs. $y / l_{v}$.

layer fig. 9 shows that friction is pointed against the flows. It rotates progressively with depth and is more and more pointed against gravity. In order to explain to understand the decrease of the effective wall friction coefficient we studied the trajectories of the grains. Fig. 10 reports the trajectory of a grains. This trajectory (the successive positions of the particle tracked in each image) has been obtained experimentally for a grain in contact with a sidewall and that belongs to the quasitatic layer where grains exhibit creeping. We clearly observe fluctuating motion that reveals a caging motion: the grains seems to be trapped in a finite area before escaping and being trapped again. The evolution of $y$ with time (Fig. 10, inset) confirms the presence of quick rearrangements between two long periods of trapping. Thus, the motion of the grains in the quasistatic layer is intermittent. This explains the very small values of stream wise average velocity measured in the quasistatic layer (from $1 \mathrm{~mm} . \mathrm{s}^{-1}$ to less than $1 \mathrm{~nm} . \mathrm{s}^{-1}$ ) [9]. Similarly to what is observed during granular compaction [18] a large motion is not a motion from one cage to another one, but a cage deformation. The quick rearrangements observed become less and less frequent as one goes deeper in the flow. While trapped there, grains describe an oscillatory motion with zero mean displacement, thus contributing negligibly to the mean resultant wall friction force $\overrightarrow{\tau_{w}}$. As trapping duration grows with depth the resultant wall friction $\mu_{\tau}$ weakens as shown in Figure 8. 


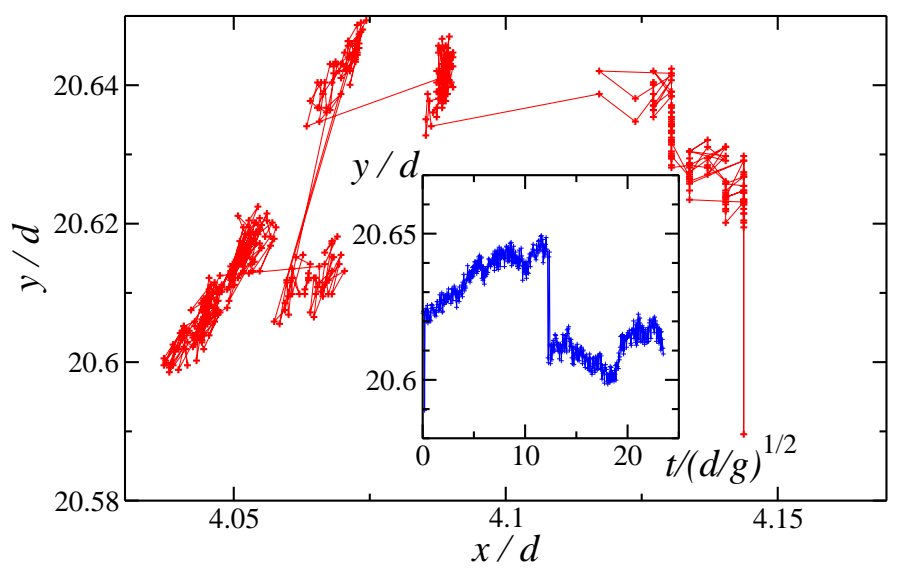

FIGURE 10. Trajectory of a grain initially located at $y / l_{v} \approx 3$. Inset : depth of the grain .vs. dimensionless time.

\section{CONCLUSION}

In this paper we show that we are able to simulate granular flows on a heap using periodic boundary conditions in the flow direction. We observe strong differences between flows on a heap and flows on a rough bottom. Our results demonstrate that intermittent motion weakens the resultant wall friction on a length scale $l_{v}$. The existence of such depth is necessary and sufficient to yield $S / N=\tan \theta-\mu_{w} h / W$, relation which, as Taberlet [1], observed, gives internal friction in terms of inclination and apparent depth $h$ of the flowing layer. In doing so, we resolved the chief paradox of their analysis, which had predicted negative friction at large depths. We also identified the origin of $\mu_{w}$.

These results open new questions on granular flows. What is the effect of the friction coefficient on the characteristic length $l_{v}$ ? Which friction coefficient dominates ? The wall-grain or the grain-grain ? The case of bumpy sidewalls should also be explored since it can correspond to natural or industrial situations.

\section{ACKNOWLEDGMENTS}

This work was supported by ANR grant (ANR-05-BLAN-0273), NSF travel grant INT-0233212 and CNRS (PICS France-USA). The authors acknowledge stimulating discussions with James Thomas Jenkins and Diego Berzi. We also thank Paul Sanchez for his preliminary work on the friction weakening.

\section{REFERENCES}

1. N. Taberlet, P. Richard, A. Valance, W. Losert, J-M. Pasini, J. T. Jenkins and R. Delannay, Phys. Rev. Lett. 91, 264301(2003).

2. P. Jop, Y. Forterre and O. Pouliquen, Journal of Fluid Mechanics 541, pp. 167-Ü192. (2005).

3. J. Rajchenbach, Phys. Rev. Lett. 65, 2221 (1990)

4. N. Taberlet, P. Richard,1 and E. J. Hinch, Phys. Rev. E 73, 050301 (2006).

5. A. Orpe and D.V. Khakhar, Phys. Rev. E 64, 031302 (2001)

6. G. Félix, V. Falk, and U. DŠOrtona, Eur. Phys. J. E 22, 25 Ü31 (2007)

7. P.-A. Lemieux and D. J. Durian, Phys. Rev. Lett. 85, 4273-4276 (2000).

8. T. S. Komatsu, S. Inagaki, N. Nakagawa, and S. Nasuno, Phys. Rev. Lett. 86, 1757 (2001).

9. J. Crassous, J-F. Metayer, P. Richard and C. Laroche, J. Stat. Mech. P03009 (2008).

10. P. Philippe and D. Bideau, Europhys. Lett. 60 (5) 677-683 (2002).

11. Ph. Ribière, P. Richard, P. Philippe, D. Bideau and R. Delannay, Eur. Phys. J. E 22 249-253 (2007)

12. B. Smit and D. frenkel, Understanding Molecular Simulation. From Algorithms to Applications, Academix Press (2001).

13. N. Taberlet N. Taberlet, P. Richard, E. Henry and R. Delannay, Europhys. Lett. 68 515-521 (2004).

14. N. Taberlet, P. Richard and Renaud Delannay, Computers \& Mathematics with Applications, 55, 230 - 234, (2008).

15. N. Taberlet, PhD thesis, university of Rennes I (2005).

16. P. Richard, A. Valance, J.F Métayer, P. Sanchez, J. Crassous, M. Louge and R. Delannay, Phys. Rev. Lett. 101, 248002 (2008). 
17. L. E. Silbert, D. Ertas, Gary S. Grest, Thomas C. Halsey, Dov Levine, and Steven J. Plimpton, Phys. Rev. E, 64, 051302 (2001).

18. P. Ribière, P. Richard, R. Delannay, D. Bideau, M. Toiya, and W. Losert, Phys. Rev. Lett., 268001 (2005).

19. J. Rajchenbach, Phys. Rev. Lett. 90, 144302 (2003). 